
$\$$ Research Square
Preprints are preliminary reports that have not undergone peer review.
They should not be considered conclusive, used to inform clinical practice,
or referenced by the media as validated information.

\title{
DAB2IP predicts treatment response and prognosis of ESCC patients and modulates its radiosensitivity through enhancing IR-induced activation of the ASK1-JNK pathway
}

\section{Yue Li}

First Affiliated Hospital of Anhui Medical University

\section{Meng Xu}

First Affiliated Hospital of Anhui Medical University

\section{Weiyang Fang}

First Affiliated Hospital of Anhui Medical University

\section{Xiangcun Chen}

First Affiliated Hospital of Anhui Medical University Jifei Xu

First Affiliated Hospital of Anhui Medical University

\section{Liang Xiao}

First Affiliated Hospital of Anhui Medical University

\section{Shuhao Pan}

First Affiliated Hospital of Anhui Medical University

\section{Lei Sheng}

First Affiliated Hospital of Anhui Medical University

\section{Huaguang Pan}

First Affiliated Hospital of Anhui Medical University

\section{Huiping Chai}

First Affiliated Hospital of Anhui Medical University

\section{Tianqi Zha}

First Affiliated Hospital of Anhui Medical University

\section{Lei Zhao}

Sun Yat-sen University Cancer Center

\section{Fan Wang}

First Affiliated Hospital of Anhui Medical University

Dan Xie

Sun Yat-sen University Cancer Center

\section{Chaozhao Liang}

First Affiliated Hospital of Anhui Medical University 


\section{Zhuting Tong ( $\square$ zhuting_tong@ahmu.edu.cn )}

First Affiliated Hospital of Anhui Medical University

\section{Research}

Keywords: Esophageal squamous cell carcinoma; DAB2IP; chemoradiosensitivity;

Posted Date: April 23rd, 2020

DOI: https://doi.org/10.21203/rs.3.rs-23979/v1

License: (c) (1) This work is licensed under a Creative Commons Attribution 4.0 International License. Read Full License 


\section{Abstract}

Background: Disabled homolog 2 interacting protein (DAB2IP) plays a tumor-suppressive role in several types of human cancers. However, the molecular status and function of the DAB2IP gene in esophageal squamous cell carcinoma (ESCC) is rarely reported.

Methods: We examined the expression dynamics of DAB2IP by immunohistochemistry (IHC) in 140 ESCC patients treated with definite chemoradiotherapy. A series of in vivo and in vitro experiments were performed to elucidate the effect of DAB2IP on the chemoradiotherapy (CRT) response and its underlying mechanisms in ESCC.

Results: Decreased expression of DAB2IP in ESCCs correlated positively with ESCC resistance to CRT and was a strong and independent predictor for short disease-specific survival (DSS) of ESCC patients. Furthermore, the therapeutic sensitivity of CRT was substantially increased by ectopic overexpression of DAB2IP in ESCC cells. In addition, knockdown of DAB2IP dramatically enhanced resistance to CRT in ESCC. Finally, we demonstrated that DAB2IP regulates ESCC cell radiosensitivity through enhancing ionizing radiation (IR)-induced activation of the ASK1-JNK signaling pathway.

Conclusions: Our data highlight the molecular etiology and clinical significance of DAB2IP in ESCC, which may represent a new therapeutic strategy to improve therapy and survival for ESCC patients. Keywords: Esophageal squamous cell carcinoma; DAB2IP; chemoradiosensitivity; ASK1; JNK

\section{Background}

Esophageal carcinoma is the eighth most common cancer and the sixth leading cause of cancer-related deaths worldwide. Esophageal squamous cell carcinoma (ESCC) is the dominant histological type of esophageal cancer in China, with a five-year overall survival less than $30 \%$.(1) At present, a definitive concurrent chemoradiotherapy (CRT) has been established as the standard treatment for advanced localized ESCC.(2) However, therapeutic outcomes are not satisfactory, even with the recent rapid development of diagnostics and therapies. Chemoradioresistance is one of the most important factors for the poor prognosis in ESCC patients.(3) Currently, the molecular mechanisms underlying chemoradioresistance in ESCC have not been clearly elucidated. Thus, it is necessary to identify novel markers that can predict responses to chemoradiotherapy, so as to find novel therapeutic targets and develop new modalities of treatment.

DAB2IP, also named ASK1-interacting protein-1 (AIP1), is a novel member of the Ras-GTPase-activating protein family.(4) DAB2IP has been identified as an EZH2 target gene by epigenetic silencing mechanism. The N-terminal domain of DAB2IP interacts with tumor suppressor DOC2/DAB2 to form a unique protein complex that has negative regulatory activity targeting the Ras-mediated signaling pathway.(5) Recently, there has been increasing evidence that reduced expression of DAB2IP occurs in several types of human malignancies, including prostate cancer,(6) lung cancer,(7) hepatocellular carcinoma,(8) renal cancer,(9) and breast cancer.(10) Loss expression of DAB2IP was observed to correlate closely with tumor 
aggressiveness and/or poor patient prognosis. Several recent studies showed that downregulation of DAB2IP expression in prostate cancer cells results in resistance to radiotherapy. $(6,11,12)$ To date, however, the molecular status of the DAB2IP gene in ESCC and the relationship between the expression of DAB2IP and chemoradiosensitivity in ESCC has not been elucidated.

Notably, our group's previous work demonstrated that EZH2, an upstream inhibitory regulatory protein of DAB2IP, serves as a promising diagnostic biomarker for hepatocellular carcinoma(13) and supports nasopharyngeal carcinoma cell aggressiveness.(14) Most importantly, we further revealed that high expression of EZH2 was correlated with a poor response to chemoradiotherapy in ESCC patients.(15) These evidences promote us to feel great interest in determining the significance of DAB2IP, as an identified repressive target gene of EZH2, in CRT sensitivity and its effect on the prognosis of ESCC patients treated with definite CRT.

In this study, to determine whether abnormalities of DAB2IP were involved in the pathogenesis and chemoradiosensitivity of ESCC, we applied immunohistochemistry (IHC) analysis to examine the expression of DAB2IP in a cohort of biopsy specimens of primary ESCC patients treated with definitive CRT. Furthermore, to examine whether the expression of DAB2IP could predict the ESCC response to CRT and patient clinical outcomes, we evaluated the correlation between DAB2IP expression and patient clinical/prognostic factors. In addition, the underlying molecular mechanisms of DAB2IP involved in chemo/radioresistance in ESCC were investigated.

\section{Methods}

\section{Cell culture, antibodies, and chemical reagents}

The three ESCC cell lines (i.e., TE1, EC109, andKyse150) were purchased in 2015 from the Cell Bank of the Chinese Academy of Sciences (Shanghai, China) where they were characterized by mycoplasma detection, DNA-Fingerprinting, isozyme detection and cell vitality detection. The ESCC cell lineKyse30 was kindly provided by Prof. Xiaofeng Zhu (Sun Yat-Sen University Cancer Center) in 2014. All cell lines were maintained in RPMI-1640 supplemented with 10\% fetal bovine serum and were authenticated three months before the beginning of the study (2015) based on viability, recovery, growth and morphology by the supplier. All the cell lines have not been in culture for greater than 2 months.

Primary antibodies used in this study: rabbit anti-DAB2IP (ab87811, Abcam, UK); rabbit anti-cleaved poly (ADP-ribose) polymerase (PARP, Asp214), rabbit anti-cleaved caspase-3 (Asp175), mouse anti-PhosphoHistone H2AX(Y-H2AX) (Ser139), rabbit anti-53BP1, rabbit anti-AKT and anti-Phospho-AKT(Ser473), rabbit anti-JNK and anti-Phospho-JNK, rabbit anti-ERK1/2 and anti-Phospho-ERK1/2, rabbit anti-ASK1 and antiPhospho-ASK1 (ser966), rabbit anti-14-3-3, rabbit anti-Thioredoxin(Cell Signaling Technology, Boston. MA); a-tubulin (BD Transduction Laboratories). Secondary antibodies: Dylight 549-conjugated goat antirabbit IgG (Proteintech Group, Inc., Chicago, IL), Alexa Fluor 488-conjugated goat anti-mouse IgG (Invitrogen, Carlsbad, CA). Peroxidase-conjugated goat anti-rabbit IgG and goat anti-mouse IgG (Santa Cruz Biotechnology, Santa Cruz, CA). Annexin V-FITC/PI apoptosis detection kit (Vazyme Biotech, China). 
Additional methods

All further methods can be found in Additional file 3 (Supplementary Materials and Methods).

\section{Results}

\section{Patient characteristics}

The clinicopathological characteristics of the 140 ESCC patients are summarized in Table1. According to the 8th edition of the TNM classification of the American Joint Committee on Cancer (AJCC)(16), 74 patients were classified into Stage II and Stage III, and 66 cases were Stage IV. All the patients received the same regimen of concurrent CRT described above. During the study period, CR and non-CR were achieved in 36 and 104 patients, respectively. The CR rate was 25.7\%. Among the 104 patients who did not achieve CR, 72 cases received adjuvant chemotherapy, and 10 cases received surgical esophagectomy. The other patients did not receive any antitumor treatments until tumor progression.

\section{Selection of cutoff score for high expression of DAB2IP}

To assess statistical significance and avoid the problems of multiple cut-point selection, $\mathrm{X}$-tile program(17) was used to determine an optimal cutoff value for expression of DAB2IP protein while correcting for the use of minimum $P$ statistics by Miller-Siegmund $P$-value correction. The calculated staining score of immunopositive cells ranged from 0 to 12 in all tissues. Based on the cut-point value determined by $\mathrm{X}$-tile software related to survival status, we categorized the samples into low (IHC score $\leq 5)$ and high (IHC score $>5$ ) expression subgroups (Figure1C-a,b).

\section{Reduced expression of DAB2IP protein in ESCC cell lines and ESCC biopsy tissues}

In this study, the protein levels of DAB2IP were examined by Western blotting (Figure1A). All the four ESCC cell lines expressed relatively lower levels of DAB2IP than non-cancerous esophageal control tissues. In addition, EC109 cells displayed the highest levels of endogenous DAB2IP expression, whereas Kyse150 cell line showed the lowest level expression (Figure1A, left panel). In 14 paired primary tissues, 10/14 (71.4\%) showed downregulated DAB2IP expression when compared with adjacent non-neoplastic esophageal tissues. The 10 representative ESCC cases with downregulated expression of DAB2IP are illustrated in Figure1A, right panels.

Next, we performed IHC to evaluate the expression of DAB2IP in the 140 ESCC biopsy specimens and 25 corresponding normal mucosal tissues. The DAB2IP staining showed weak and diffuse immunostaining in background stromal cells. Positive signals of DAB2IP protein were predominantly located in the cytoplasm of esophageal cell and also weakly stained in the nucleus. According to the criteria mentioned above, low expression of DAB2IP was observed in 105/140(75.0\%) of primary ESCCs biopsy tissues compared with only $2 / 25(8 \%)$ in normal esophageal mucosa (Table1).

\section{Correlation of DAB2IP expression with clinicopathologic variables}


Table 1 summarizes the detailed information about the rates of low expression of DAB2IP with respect to several standard clinicopathological features in the cohort. Results showed that no significant association was found between low DAB2IP expression and some of the clinicopathological features, such as patient's age, gender, and tumor location( $P>0.05)$. Interestingly, DAB2IP expression correlated closely with World Health Organization (WHO) grade $(P<0.001)$, Tumor size $(P<0.001)$, T status $(P=$ $0.003)$, Node $(\mathrm{N})$ status $(P<0.001)$, and distant lymph node metastasis $(M)$ status $(P=0.017)$ (Table1).

\section{Relationship between DAB2IP expression and CRT response}

Primary CR was achieved in 25.7\% (36/140) of the patients with ESCC. Moreover, using the optimal cutoff value of $>5$ score, DAB2IP expression was also the factor that showed a significant correlation with CRT response in the cohort $\left(r_{p}=0.322, n=140, P<0.001\right)$ in which low expression of DAB2IP was observed more frequently in the non-CR subset than the CR subset.

\section{Correlation between clinicopathological variables, DAB2IP expression and ESCC patient survival}

Of the 140 patients with ESCC, 8 patients were lost to follow-up. The median survival time was 17.8 months. The 2-year OS and DSS for the entire cohort of patients were $38.6 \%$ and $36.6 \%$, respectively. Kaplan-Meier analysis revealed that low-level expression of DAB2IP was associated with short overall survival time $(P=0.002)$ and disease specific survival time $(P<0.001)$ (Figure1C-c, d).

In log-rank test, low DAB2IP expression was also found to correlate closely with poor DSS of ESCC patients (median14.77 versus 34.50months, $P<0.001$ ) (Table 2). Further univariate Cox regression analysis identified WHO grade, tumor size, T status, $\mathrm{N}$ status, $\mathrm{M}$ status, $\mathrm{CRT}$ response and DAB2IP expression to have a significant impact on DSS $(P=0.012,0.003,0.001,0.030,0.022,<0.001$ and $<0.001$ respectively; Table 3$)$. Other clinicopathological variables, including age, gender and location showed no significant correlation with DSS ( $P=0.908,0.317$ and 0.387 respectively; Supplementary 2$)$. The parameters that were significant in univariate analysis were further evaluated in Cox regression multivariate analysis. The results showed that the expression of DAB2IP $(P<0.001)$, T status $(P<0.001)$, and CRT response $(P=0.004)$ were independent predictors of tumor prognosis ((Additional file 1: Table S1).

\section{DAB2IP regulates the chemosensitivity of ESCC cells to cisplatin in vitro}

To further investigate whether DAB2IP is capable of modulating the chemoresistance of ESCC cells in vitro, the Kyse150 cell line, which is showed the lowest endogenous expression levels of DAB2IP, was subsequently transfected with pcDNA3.1-DAB2IP or the control plasmid pcDNA3.1. As shown in Figure2A left, ectopic expression of DAB2IP in Kyse150 cells (Figure2C, left) resulted in a substantial reduction of $\mathrm{IC}_{50}$ value under cisplatin treatment compared to that of the empty vector control cells (5.52uMvs 10.97uM). Consistently, stably silencing DAB2IP by lentivirus in EC109 cells, which showed a relative high expression of DAB2IP (Figure 1A, left panel), resulted in increased $\mathrm{IC}_{50}$ value compared with the control 
EC109-shluc cells (1.35uMvs 2.78uM, Figure 2A right). The knockdown efficiency of shRNA lentivirus was validated by Western blot (Figure $2 \mathrm{C}$, right).

To further identify the crucial role of DAB2IP in ESCCs chemoresistance, the Annexin V/PI assay was performed to evaluate the effect of DAB2IP on apoptosis. As illustrated in Figure 2, the proportion of apoptosis was dramatically increased when Kyse150-DAB2IP cells were pretreated with cisplatin(25.560 $\pm 4.680 \%$ vs $38.160 \pm 5.620 \%, P=0.041$, Figure $2 \mathrm{~B}$, upper panels). Accordantly, ablation of endogenous DAB2IP remarkably reduced cisplatin-induced apoptosis in EC109 cells $(32.840 \pm 4.57 \%$ vs $21.280 \pm 4.890 \%, p=0.0403$, Figure 2B, lower panels).

In agreement with the results obtained by Annexin V/PI assay, our following Western blot analysis indicated that the ectopic expression of DAB2IP in Kyse150 cells obviously increased protein levels of cleaved-caspase 3 and cleaved-PARP induced by cisplatin (Figure $2 \mathrm{C}$ left). On the other hand, the depletion of endogenous DAB2IP in EC109 cells led to a substantial reduction of cleaved-caspase 3 and cleaved-PARP in the presence of cisplatin (Figure $2 \mathrm{C}$ right). Taken together, these results provide strong evidence that DAB2IP plays a crucial role in regulating chemosensitivity of ESCC cells.

\section{DAB2IP influences the sensitivity of ESCC cells to ionizing radiation (IR) in vitro}

Since our above ESCC cohort data revealed that DAB2IP expression was significantly correlated with CRT response (Table1), we next used clonogenic assay to determine the important role of DAB2IP in ESCC radiosensitivity in vitro. As shown in Figure 3A, ectopic overexpression of DAB2IP in Kyse150 cells resulted in an obviously reduction of clonogenic survival fractions when compared with the corresponding control Kyse150-vector cells (Figure 3A left). In addition, virtually increment of clonogenic survival fractions was observed in DAB2IP repressed EC109 cells in comparison to control EC109-shluc cells. Additional non-linear curve-fitting analysis of the dose-response survival curves was performed according to the linear quadratic (LQ) model, and the parameters for each curve including a value, $\beta$ value, $\alpha / \beta$ value, SF2, and sensitizing enhancement ratio (SER) were listed (Additional file 2: Table $S$ 2). According to these values, SF2 was markedly reduced in Kyse150-DAB2IP cells compared to the control Kyse150-vecter cells ( 0.43 vs 0.66 ) with consequence that SER value was 1.52 . Consistently, a substantial increase of SF2 value was observed in DABIP-silenced EC109 cells when compared with control EC109-shluc cells (0.67 vs 0.59), which indicated that the SER of DAB2IP silencing in EC109 cells was 0.87 (Additional file 2: Table S2).

Next, to obtain more evidence regarding the involvement of DAB2IP protein in regulating ESCC cell radiosensitivity, Annexin V/PI assay and immunoblots for detecting the apoptosis-related marker proteins (i.e., cleaved caspase-3 and cleaved PARP) was perfomed. As shown in Figure 3B and 3C, with the absence of IR, neither overexpression nor knockdown of DAB2IP could influence the proportion of apoptosis and the levels of apoptosis-related marker proteins. This phenomenon had changed after treating cells with 3Gy X-rays. In the presence of IR, both Annexin V/PI assay and Western blot results indicated that the apoptotic activity of DAB2IP was significantly enhanced in DAB2IP-overexpressed Kyse150 cells in contrast to control Kyse150-vector cells, whereas repression DAB2IP in EC109 cells 
cause a remarkable decrease of apoptotic cells $(32.360 \pm 5.210 \% \mathrm{vs} 17.890 \pm 4.65 \%, P=0.023)$, cleaved caspase-3, and cleaved PARP when compared with corresponding control EC109-shLuc cells.

\section{The radiosensitizing activity of DAB2IP is associated with enhanced double-stranded DNA breaks (DSB) repair in ESCC cells}

It is well known that the DSB repair capacity is closely correlated with intrinsic radiosensitivity of cells. (18) DSB repair involves the rapidly phosphorylation of $\mathrm{YH} 2 \mathrm{AX}$ and the $\mathrm{P} 53$ binding protein (53BP1) recruitment in site-specific DNA damage.(19) Thus, the formation of $\mathrm{y}-\mathrm{H} 2 \mathrm{AX}$ and 53BP1 foci, which displayed as discrete foci at surrounding DNA double-strand breaks, is widely used to monitor radiationinduced DNA breaks and to assay DNA rejoining defects.(20)

To investigate whether DAB2IPcaninfluence DNA repair ability of ESCC cells, the dual immunofluorescence staining for 53BP1 (red) and phospho-yH2AX (green) foci was performed. The DSB repair kinetics were determined by counting the colocalized foci (yellow). As illustrated in Figure 3D upper panels, after $36 \mathrm{~h}$ of IR treatment, the unrepaired DNA damage detected by counting colocalized foci of YH2AX and 53BP1 was significantly increased in Kyse150-DAB2IP cells when compared with control Kyse150-vector cells ( $97 \pm 18$ vs $206 \pm 22, P=0.003$ ), while no obvious difference was observed before IR treatment (0h). Consistently, 36 hours after IR exposure, the colocalized foci number of YH2AX and 53BP1 was remarkably reduced in DAB2IP-silenced EC109 cells when compared to control EC109-shluc cells(388 $\pm 36 v s 246 \pm 38, p=0.038)$. However, without IR treatment (Oh), no distinct difference was detected in these two group cells. These data indicated that the expression of DAB2IP was associated with DSB repair capability of ESCC cells.

\section{Overexpression of DAB2IP enhances the radiosensitivity of esophageal squamous cell carcinoma cells in vivo}

To further determine whether DAB2IP has similar impact on ESCC cell response to IR in vivo, Kyse150DAB2IP and control Kyse150-vector cells were inoculated into female BALB/c nude mice. In agreement with our in vitro experiments, we found that overexpression of DAB2IP alone did not influence tumorigenicity of ESCC cells, (i.e., both the injected Kyse150-DAB2IP and control Kyse150-vector cells showed a similar efficiency and growth rate of the ESCC xenografts in nude mice)(Figure 4A).However, when the mice-bearing tumors received 6Gy of IR, the growth of the Kyse150-DAB2IP tumors was significantly inhibited (from a mean tumor volume of $180 \mathrm{~mm}^{3}$ before IR treatment, increasing to $287.00 \pm 38.00 \mathrm{~mm}^{3}$ at the endpoint of observation). In comparison, the increase in tumor volume of control Kyse150-vector was greater, from a mean tumor volume of $180 \mathrm{~mm}^{3}$ to $\left.423.00 \pm 43.00 \mathrm{~mm}^{3}\right)(P$ $<0.01$; Figure $4 \mathrm{~A}$ ).

The stably overexpressed levels of DAB2IP measured by IHC staining in Kyse150 xenograft tumors are shown in Figure 4B. Similar to the results observed in above in vitro experiment, the in vivo radiationinduced $\mathrm{YH} 2 \mathrm{AX}$ foci assay indicated that, 24 hours after receiving $6 \mathrm{~Gy}$ radiation, the unrepaired DNA 
damage was substantially increased in Kyse150-DAB2IP xenograft tumor tissues compared to parental control cell line xenograft tumors (Figure 4C).

Collectively, the in vivo and in vitro data demonstrated that DAB2IP plays a crucial role in modulating radiosensitivity of ESCC cells.

\section{DAB2IP regulates ESCC cell radiosensitivity, possibly through enhancing IR-induced activation of ASK1- JNK signaling}

Apoptosis is an important mechanism by which IR exerts its therapeutic response and faulty apoptosis is a known mechanism leading to resistance to radiation therapy.(21) In previous literature, DAB2IP has been demonstrated to be involved in several apoptotic pathways, including Ras/Raf/MEK/ERK (MAPK) signaling, PI3K/Akt signaling and ASK1-JNK signaling.(22-24) Therefore, to address which signaling pathways were critically involved in DAB2IP regulating ESCC cells radiosensitivity, we initially performed Western blot to evaluate the phosphorylation levels of AKT, JNK, and ERK, which represent the activated degree of these three signaling pathways, respectively. As shown in Figure $5 \mathrm{~A}$, in the present of $3 \mathrm{~Gy} \mathrm{IR}$ treatment, stably silencing DAB2IP by lentivirus in EC109 cells obviously increased the protein levels of $p$ JNK. No changes were observed in p-ERK and p-AKT levels. This result implied that ASK1-JNK signaling pathway might be an important mechanism in DAB2IP regulating ESCC cells radiosensitivity.

\section{DAB2IP facilitates IR-induced dephosphorylation at Ser-966 of ASK1 accompanied by reinforce dphosphorylation of JNK}

Next, to confirm whether ASK1-JNK pathway was involved in DAB2IP-inducedregulation of ESCC cells radiosensitivity, the stably ectopic DAB2IP-overexpressed Kyse150 cells were established and followed by 3Gy X-ray irradiation. The dephosphorylated levels of ASK1 at Ser966 and phosphorylated levels of JNK, both triggered by X-ray irradiation, were determined by Western blot. As illustrated in Figure 5B, elevated expression of DAB2IP alone in Kyse150 cells did not alter p-ASK1ser966 and p-JNK levels in the absence of IR treatment. However, when treated cells with 3Gy IR, ectopic overexpression of DAB2IP remarkably reduced the levels of p-ASK1-ser966, concomitant with a substantially increased levels of p-JNK. These observations were further supported by our results from knockdown experiments. As anticipated, silencing DAB2IP by lentivirus alone in EC109 cells did not affect the protein levels of ASK1 p-Ser966 and p-JNK in the condition without IR. However, in the presence of IR, we observed dramatically increased levels of p-ASK1 Ser966 concurrently with virtual reduction of p-JNK in DAB2IP-depleted EC109 cells when compared with our control EC109-shluc cells (Figure 5C).

\section{DAB2IP enhances IR-induced ASK1 activation by facilitating dissociation of ASK1 from its inhibitor 14-3- 3 but not from its other inhibitor, thioredoxin (Trx)}

It has been well documented that 14-3-3 is one of the most important inhibitors of ASK1.The dephosphorylation at Ser-966 of human ASK1 and consequent dissociation of 14-3-3 with ASK1 has been thought to be a crucial activation mechanism of ASK1-JNK pathway.(25-28) In addition, it was 
further reported that DAB2IP could enhance TNF- $a$-induced ASK1 activation by facilitating dissociation of inhibitor 14-3-3 from ASK1.(29) Thus, we posited that DAB2IP might be capable of intensifying IRinduced ASK1 activation through a similar mechanism. To test this premise, Kyse150 cells were firstly transfected with vector pcDNA3.1 or pcDNA3.1-DAB2IP plasmid, then cells were treated with or without IR, and the association of ASK1 with 14-3-3 was determined by immunoprecipitation with anti-ASK1 followed by Western blot with anti-14-3-3. As expected, the interaction between ASK1 and 14-3-3 was substantially reduced in response to IR treatment, whereas this reduction was reinforced by elevated expression of DAB2IP (Figure 5D).Accordingly, the radiation-induced disruptive effect on the ASK1-14-3-3 complex was also observed in EC109 cells, and this disruptive effect was markedly attenuated by knocking down of DAB2IP (Figure 5E).

Besides, we also determined whether DAB2IP could affect IR-induced dissociation of ASK1 with Trx, another important negative regulator of ASK1. Intriguingly, our coimmunoprecipitation assay showed that neither knockdown nor overexpression of DAB2IP could impact on IR-induced disruption of ASK1-Trx complex (Figure 5D and 5E). This important result has excluded the possibility that DAB2IP regulated IRinduced ASK1 activation by enhancing the dissociation of ASK1 with Trx.

\section{The enhanced activity of DAB2IP on IR-activated JNK signals dependent on the presence of ASK1}

In order to further identify whether the sensitizing effect of DAB2IP in response to IR was through ASK1JNK pathway, Kyse150 cells were firstly stably knocked down ASK1 by lentivirus, and subsequently, the control pcDNA3.1 or pcDNA3.1-DAB2IP plasmid was transfected into these cells to determine whether DAB2IP can still activate JNK signal in the absence of ASK1. As shown in Figure $5 F$, in the presence of IR, the enhanced activity of DAB2IP toward JNK signal was virtually totally prevented in ASK1-depleted Kyse 150 cells. These data suggested that the presence of ASK1 was required and might be a predominant mediator for the enhanced activity of DAB2IP toward JNK signal.

\section{Discussion}

It is well known that definitive CRT is a standard treatment for locally advanced ESCC patients, and CRT response is widely accepted as one of the most important prognostic factors of ESCC. $(30,31)$ However, the response of individual tumors to CRT are varies widely. Thus, investigation of specific molecular markers that might serve as predictors for CRT response, as well as prognostic factors in ESCC, are highly desirable and have long been sought.

$D A B 2 I P$, also known as ASK-interacting protein 1, is a potential tumor suppressor gene that is capable of modulating the cytoplasmic steps of various oncogenic pathways, including proliferation, survival, and apoptosis. $(9,23)$ Loss of DAB2IP expression is frequently observed and is associated with aggressiveness, therapeutic resistance, and poor prognosis in various human cancers. $(4,9,10)$ However, the expression profile of DAB2IP and its effect on chemoradiotherapy sensitivity in ESCC are still unclear. To investigate the potential oncogenic role of DAB2IP in ESCC, we initially examined the expression level of the DAB2IP protein in a series of paired ESCCs with the adjacent non-neoplastic tissues. Our Western 
blot results showed that 10/14 (71.43\%) of ESCCs exhibited lower expression of DAB2IP than that in adjacent non-neoplastictissues. Next, we further examined the protein expression of DAB2IP by IHC in 25 normal esophageal mucosa tissues and 140 ESCC cases with complete follow-up information. Consistently, we observed that most of the ESCC specimens (105/140, 75.0\%) displayed low expression levels of DAB2IP compared with the only $2 / 25(8 \%)$ in normal esophageal mucosal tissues. Further correlation analyses revealed that low expression of DAB2IP was closely correlated with poor response to CRT. Moreover, decreased DAB2IP expression was a strong and independent predictor for poor survival of ESCC patients treated with definitive CRT. Taken together, these results suggested a potential impact of DAB2IP on the cellular responses to ionizing radiation and cytotoxic drugs in ESCC.

Subsequently, a series of in vitro and in vivo experiments was performed to confirm the crucial role of DAB2IP in regulating ESCC chemoradiosensitivity. Our observation indicated that ectopic overexpression of DAB2IP significantly enhanced the sensitivity of ESCC cells to IR and cisplatin by inducing more apoptosis. This conclusion was further supported by subsequent knockdown experiments. It is worthwhile to note that, without IR treatment, neither overexpression nor knockdown of DAB2IP could affect the proportion of apoptosis and the levels of apoptosis-related marker proteins. These results indicated that DAB2IP might be a promising chemosensitizer or radiosensitizer in ESCC therapy. In agreement with our findings, Wu et al.(32) revealed that loss of DAB2IP expression enhanced chemoresistance in CRPC (castration-resistant prostate cancer) cells through regulation ofEgr-1/Clusterin signaling network. Another study further elucidated that reduced expression of DAB2IP confers a survival advantage in prostate cells receiving IR exposure due to enhanced DSB repair, and resistance to IRinduced apoptosis.(6) Thus, these data, combined with our findings, implied that DAB2IP might be a potentially promising chemo/radiosensitizer that can improve strategies to overcome traditional drug or radiotherapy resistance in human cancers.

Apoptosis is a distinct type of cell death, which can be induced by IR.(21) The intrinsic susceptibility to radiation-induced apoptosis is one of the most important factors that can determine tumor radiosensitivity. It has been well reported that DAB2IP,as a putative apoptosis regulator, could facilitate cell apoptosis via several apoptotic pathways, including Ras/Raf/MEK/ERK (MAPK) signaling,(22) $\mathrm{PI3K} /$ Akt signaling,(23) and ASK1-JNK signaling.(24) Thus, to determine which pathway was responsible for DAB2IP regulation of ESCC cell radiosensitivity, we compared the phosphorylation levels of AKT, JNK, and ERK between EC109-shluc and EC109-shDAB2IP cells after 3Gy IR exposure. Significantly, our Western blot results showed that p-JNK was reduced in DAB2IP-silenced EC109 cells, suggesting that the ASK1-JNK pathway might be a crucial mechanism involved in DAB2IP modulation of the radiosensitivity of ESCC cells.

Apoptosis signal-regulating kinase 1 (ASK1) was initially identified as a MAP3K, and it selectively activates the c-Jun N-terminal kinase (JNK) and p38 pathways in response to various stimuli.(33) One of the most important activators of ASK1 is oxidative stress. Under normal physiological conditions, ASK1 is maintained in an inactive state through binding to the inhibitory factors thioredoxin (Trx) and 14-3-3 proteins. $(25,34)$ In response to ROS stimulation, Trx is oxidized and dissociated from ASK1. This ROS- 
induced dissociation of Trx may initiate a conformational change in ASK1, exposing phosphorylated serine residue site (Ser-966 in humans) to a bound serine phosphatase. The phosphorylation of Ser-966 causes the release of 14-3-3 proteins from ASK1, which might finally lead to ASK1 activation. $(28,35)$

To elucidate the detailed mechanism of the ASK1-JNK pathway involvement in DAB2IP regulation of ESCC radiosensitivity, a series of experiments were conducted. Unexpectedly, our Western blot results indicated that ectopic overexpression of DAB2IP alone did not induce the dephosphorylation of Ser-966, and release of 14-3-3 from ASK1, as well as activation of JNK pathways. However, when cells were treated with 3Gy IR, overexpression of DAB2IP obviously enhanced the IR-induced dephosphorylation of Ser-966, leading to increased dissociation of ASK1 from 14-3-3 and the subsequent augmented activation of the JNK pathway. These results suggested that IR might remove the inhibitory factors that are required for the enhanced activation function of DAB2IP on ASK1-JNK signaling.

It is well established that IR can either directly or indirectly generate intracellular reactive oxygen species (ROS), which function as a key intermediates in the radiation mediated induction of apoptotic signaling in tumor cells.(36-38) As we mentioned above, when Trx is oxidized by ROS, it releases from ASK1, permitting ASK1 to be dephosphorylated on Ser-966 by ROS, which, in turn, induces dissociation of ASK1 from its inhibitor protein $14-3-3$, ultimately leading to activation of the ASK1-JNK pathway. $(25,28,34,35)$ Accordingly, our immunoprecipitation results showed that IR indeed triggers dissociation of ASK 1 from both 14-3-3 and Trx (Figure 5D and 5E). This step is necessary for basic activation of ASK1 but not sufficient for intensive ASK1 activation. It is conceivable that IR induces release of 14-3-3 and Trx in a coordinated fashion, permitting DAB2IP to further intensify ASK1 activation. Notably, we observed that only the dissociation of ASK1 from 14-3-3, but not Trx, can be enhanced by DAB2IP. This result excludes the possibility that DAB2IP enhances IR-induced ASK1 activation by facilitating the dissociation of ASK1 from Trx (Figure 5D and 5E).

Additionally, one previous important study demonstrated that, with the stimulation of TNF-a, DAB2IP preferentially binds to the locus surrounding the Ser-966-dephosphorylated site of ASK1and facilitates dissociation of 14-3-3 through recruitment of the okadaic acid-sensitive phosphatase PP2A to the ASK1 complex, which ultimately leads to the enhanced activation of JNK. $(29,39)$ In agreement with this previous result, our present study also found that DAB2IP could enhance IR-induced dephosphorylation of ASK1-Ser-966, which in turn, facilitates 14-3-3 release from ASK1, ultimately leading to intensive activation of ASK1 (Figure 5B and 5D).

From our collective data $\llbracket$ we propose a possible model to illustrate the mechanism of DAB2IP-mediated regulation of radiosensitivity of ESCC cells: (1) In ESCC cells with low or no expression of DAB2IP, IR causes increased levels of intracellular reactive oxygen species (ROS), leading to the dephosphorylation of Ser-966 of ASK1, which consequently triggers dissociation of Trx and 14-3-3 from ASK1, ultimately resulting in a basic activated status of the ASK1-JNK pathway and causing ESCC cells to exhibit low sensitivity/or resistance to radiotherapy (Figure 6G, right). (2) In ESCC cells with normal or overexpression of DAB2IP, the IR-induced ROS stimulates dephosphorylation of Ser-966 of ASK1, thereby releasing of 14-

Page 12/30 
3-3 and Trx from ASK1. This step is necessary but not sufficient for ASK1-enhanced activation. After 14-33 and Trx are released from ASK1, DAB2IP preferentially binds to the locus surrounding the Ser966dephosphorylated site of ASK1, which consequently facilitates dissociation of 14-3-3 from ASK1 and enhances ASK1 kinase activity, ultimately leading to an over-activation of ASK1-JNK signaling and ESCC cells with high sensitivity to radiotherapy (Figure 6G, left).From this model, we can easily explain the functional results observed in Figure 3 and Figure 4. That is, without IR treatment, the apoptotic activity of ASK1 was completely repressed by its inhibitors Trx and 14-3-3; thus, without IR treatment, overexpressed DAB2IP per se was incapable of inducing cell apoptosis or suppressing the tumorigenicity of ESCC cells in nude mice (Figure 3B, C and Figure 4A). These results also support the evidence that the release of Trx and 14-3-3 triggered by IR was required for the enhanced killing effects of DAB2IP.

Since the mechanisms by which IR kills cells are very complicated and still poorly understood, it is possible that other unknown pathways might also involve in DAB2IP modulation of the radiosensitivity of ESCC cells. A schematic representation of the potential molecular mechanisms underlying DAB2IP's effects in ESCC radiosensitivity is provided in Figure 5G. Clearly, further studies are needed to confirm this model and elucidate whether other pathways might also contribute to DAB2IP-mediated regulation of the radiosensitivity of ESCC cells.

In summary, our study described, for the first time, the protein expression of DAB2IP in normal human esophageal tissues and in ESCCs .In addition, our results provide a basis for the concept that downregulation of the expression DAB2IP, as detected by IHC, may be a useful tool for predicting CRT resistance and an independent prognostic factor of patients with ESCC treated with definitive CRT. We further demonstrated that the sensitizing effect of DAB2IP in response to IR is through enhanced activation of the ASK1-JNK pathway. These findings might be helpful in clinical practice for selecting potential radioresistant ESCC patients who might require a more intensive follow-up and/or more aggressive treatment.

\section{Conclusion}

Our present study firstly found that DAB2IP is low-expressed in primary ESCC biopsy samples and is closely correlated with a poor response to CRT. Further mechanistic investigations revealed that the radiosensitizing effect of DAB2IP occurs through enhanced IR-induced activation of the ASK1-JNK pathway, providing a novel strategy to overcome radioresistance in the clinical treatment of ESCC patients.

\section{Abbreviations}

DAB2IP: Disabled homolog 2 interacting protein; ESCC: esophageal squamous cell carcinoma; IHC: immunohistochemistry staining; CRT: chemoradiotherapy; IR: ionizing radiation; CR: Complete Response; OS: overall survival; DSS: disease-specific survival; SER: sensitizing enhancement ratio; 53BP1: the P53 binding protein; Trx: thioredoxin; JNK: Jun N-terminal kinase; ERK: extracellular regulated protein kinases; 


\section{Declarations}

\section{Authors' contributors}

Zhuting Tong: Conceptualization, Methodology, Software. Chaozhao Liang: Methodology, Software, Supervision. Yue Li: Investigation, Methodology. Meng Xu: Software, Writing - Original Draft. Weiyang Fang: Investigation, Writing - Original Draft. Xiangcun Chen: Resources, Data Curation. Jifei Xu: Data Curation. Liang Xiao: Data Curation. Shuhao Pan: Investigation. Lei Sheng: Investigation. Huaguang Pan: Software. Huiping Chai: Software. Tianqi Zha: Software. Lei Zhao: Resources. Fan Wang: Supervision. Dan Xie: Supervision. All of the authors discussed the results and reviewed the manuscript.

\section{Acknowledgments:}

We thank Dr. Jiabing Fan (University of California, Los Angeles) for his valuable comments and extensive editing of the manuscript. The authors thank LetPub (www.letpub.com) for its linguistic assistance during the preparation of this manuscript.

\section{Funding}

This work was supported by the National Natural Science Foundation of China (81201743, and 81630019), the Postdoctoral Science Foundation Funded Project of Anhui Province (2015B053), and the Doctoral Fund of the Ministry of Education of China (20123420120008).

\section{Availability of data and materials}

All data presented within the article and its supplementary information are available upon request from the corresponding author.

\section{Ethics approval and consent to participate}

All patients authorized the use of their specimens by written informed consent. This study was approved by the Institute Research Medical Ethics Committee of Anhui Medical University (Hefei, China).

\section{Consent to publication}

Not applicable.

\section{Competing interests}

The authors declare no conflict of interest.

\section{Author details}

${ }^{1}$ Department of Radiation Oncology, the First Affiliated Hospital of Anhui Medical University, Hefei, China.

${ }^{2}$ Department of thoracic surgery, the First Affiliated Hospital of Anhui Medical University, Hefei, China. 
${ }^{3}$ Department of Radiation Oncology, Sun Yat-Sen University Cancer Center, Guangzhou, China. ${ }^{4}$ State Key Laboratory of Oncology in South China; Collaborative Innovation Center for Cancer Medicine; Department of Pathology, Sun Yat-Sen University Cancer Center, Guangzhou, China. ${ }^{5}$ Department of Surgery, the First Affiliated Hospital of Anhui Medical University, Hefei, Anhui, China.

Publisher's note Springer Nature remains neutral with regard to jurisdictional claims in published maps and institutional affiliations.

\section{References}

1. Bray F, Ferlay J, Soerjomataram I, Siegel RL, Torre LA, Jemal A. Global cancer statistics 2018 : GLOBOCAN estimates of incidence and mortality worldwide for 36 cancers in 185 countries. CA: a cancer journal for clinicians. 2018;68(6):394-424 doi 10.3322/caac.21492.

2. Zheng H, Ren W, Pan X, Zhang Q, Liu B, Liu S, et al. Role of intravoxel incoherent motion MRI in early assessment of the response of esophageal squamous cell carcinoma to chemoradiotherapy: A pilot study. Journal of magnetic resonance imaging : JMRI. 2018;48(2):349-58 doi 10.1002/jmri.25934.

3. Nakajima $\mathrm{M}$, Kato $\mathrm{H}$. Treatment options for esophageal squamous cell carcinoma. Expert opinion on pharmacotherapy. 2013;14(10):1345-54 doi 10.1517/14656566.2013.801454.

4. Chen $\mathrm{H}$, Pong RC, Wang Z, Hsieh JT. Differential regulation of the human gene DAB2IP in normal and malignant prostatic epithelia: cloning and characterization. Genomics. 2002;79(4):573-81 doi 10.1006/geno.2002.6739.

5. Wang Z, Tseng CP, Pong RC, Chen H, McConnell JD, Navone N, et al. The mechanism of growthinhibitory effect of DOC-2/DAB2 in prostate cancer. Characterization of a novel GTPase-activating protein associated with N-terminal domain of DOC-2/DAB2. The Journal of biological chemistry. 2002;277(15):12622-31 doi 10.1074/jbc.M110568200.

6. Kong Z, Xie D, Boike T, Raghavan P, Burma S, Chen DJ, et al. Downregulation of human DAB2IP gene expression in prostate cancer cells results in resistance to ionizing radiation. Cancer research. 2010;70(7):2829-39 doi 10.1158/0008-5472.CAN-09-2919.

7. Yano M, Toyooka S, Tsukuda K, Dote H, Ouchida M, Hanabata T, et al. Aberrant promoter methylation of human DAB2 interactive protein (hDAB2IP) gene in lung cancers. International journal of cancer. 2005;113(1):59-66 doi 10.1002/ijc.20531.

8. Qiu GH, Xie H, Wheelhouse N, Harrison D, Chen GG, Salto-Tellez M, et al. Differential expression of hDAB2IPA and hDAB2IPB in normal tissues and promoter methylation of hDAB2IPA in hepatocellular carcinoma. Journal of hepatology. 2007;46(4):655-63 doi 10.1016/j.jhep.2006.11.012.

9. Zhou J, Luo J, Wu K, Yun EJ, Kapur P, Pong RC, et al. Loss of DAB2IP in RCC cells enhances their growth and resistance to mTOR-targeted therapies. Oncogene. 2016;35(35):4663-74 doi 10.1038/onc.2016.4.

10. Ji W, Li Y, He Y, Yin M, Zhou HJ, Boggon TJ, et al. AlP1 Expression in Tumor Niche Suppresses Tumor Progression and Metastasis. Cancer research. 2015;75(17):3492-504 doi 10.1158/0008-5472.CAN- 
15-0088.

11. Yu L, Tumati V, Tseng SF, Hsu FM, Kim DN, Hong D, et al. DAB2IP regulates autophagy in prostate cancer in response to combined treatment of radiation and a DNA-PKcs inhibitor. Neoplasia. 2012;14(12):1203-12.

12. Chang R, He H, Mao G, Kong Z. Upregulating DAB2IP expression via EGR-1 inhibition, a new approach for overcoming fractionated-irradiation-induced cross-tolerance to ionizing radiation and mitomycin C in tumor cells. International journal of radiation biology. 2017;93(4):386-93 doi 10.1080/09553002.2016.1257831.

13. Cai MY, Tong ZT, Zheng F, Liao YJ, Wang Y, Rao HL, et al. EZH2 protein: a promising immunomarker for the detection of hepatocellular carcinomas in liver needle biopsies. Gut. 2011;60(7):967-76 doi 10.1136/gut.2010.231993.

14. Tong ZT, Cai MY, Wang XG, Kong LL, Mai SJ, Liu YH, et al. EZH2 supports nasopharyngeal carcinoma cell aggressiveness by forming a co-repressor complex with HDAC1/HDAC2 and Snail to inhibit E-cadherin. Oncogene. 2012;31(5):583-94 doi 10.1038/onc.2011.254.

15. He LR, Liu MZ, Li BK, Jia WH, Zhang Y, Liao YJ, et al. High expression of EZH2 is associated with tumor aggressiveness and poor prognosis in patients with esophageal squamous cell carcinoma treated with definitive chemoradiotherapy. International journal of cancer. 2010;127(1):138-47 doi $10.1002 / \mathrm{ijc} .25031$.

16. Donohoe CL, Phillips AW. Cancer of the esophagus and esophagogastric junction: an 8(th) edition staging primer. J Thorac Dis. 2017;9(3):E282-E4 doi 10.21037/jtd.2017.03.39.

17. Camp RL, Dolled-Filhart M, Rimm DL. X-tile: a new bio-informatics tool for biomarker assessment and outcome-based cut-point optimization. Clinical cancer research : an official journal of the American Association for Cancer Research. 2004;10(21):7252-9 doi 10.1158/1078-0432.CCR-040713.

18. Sak A, Stuschke M. Use of gammaH2AX and other biomarkers of double-strand breaks during radiotherapy. Seminars in radiation oncology. 2010;20(4):223-31 doi 10.1016/j.semradonc.2010.05.004.

19. Schultz LB, Chehab NH, Malikzay A, Halazonetis TD. p53 binding protein 1 (53BP1) is an early participant in the cellular response to DNA double-strand breaks. The Journal of cell biology. 2000;151(7):1381-90.

20. Burma S, Chen BP, Murphy M, Kurimasa A, Chen DJ. ATM phosphorylates histone H2AX in response to DNA double-strand breaks. The Journal of biological chemistry. 2001;276(45):42462-7 doi 10.1074/jbc.C100466200.

21. Meyn RE, Milas L, Ang KK. The role of apoptosis in radiation oncology. International journal of radiation biology. 2009;85(2):107-15 doi 10.1080/09553000802662595.

22. Min J, Zaslavsky A, Fedele G, McLaughlin SK, Reczek EE, De Raedt T, et al. An oncogene-tumor suppressor cascade drives metastatic prostate cancer by coordinately activating Ras and nuclear factor-kappaB. Nature medicine. 2010;16(3):286-94 doi 10.1038/nm.2100. 
23. Xie D, Gore C, Zhou J, Pong RC, Zhang H, Yu L, et al. DAB2IP coordinates both PI3K-Akt and ASK1 pathways for cell survival and apoptosis. Proceedings of the National Academy of Sciences of the United States of America. 2009;106(47):19878-83 doi 10.1073/pnas.0908458106.

24. Zhang H, Zhang R, Luo Y, D'Alessio A, Pober JS, Min W. AIP1/DAB2IP, a novel member of the RasGAP family, transduces TRAF2-induced ASK1-JNK activation. The Journal of biological chemistry. 2004;279(43):44955-65 doi 10.1074/jbc.M407617200.

25. Zhang L, Chen J, Fu H. Suppression of apoptosis signal-regulating kinase 1-induced cell death by 143-3 proteins. Proceedings of the National Academy of Sciences of the United States of America. 1999;96(15):8511-5.

26. Cockrell LM, Puckett MC, Goldman EH, Khuri FR, Fu H. Dual engagement of 14-3-3 proteins controls signal relay from ASK2 to the ASK1 signalosome. Oncogene. 2010;29(6):822-30 doi 10.1038/onc.2009.382.

27. Liu Y, Yin G, Surapisitchat J, Berk BC, Min W. Laminar flow inhibits TNF-induced ASK1 activation by preventing dissociation of ASK1 from its inhibitor 14-3-3. The Journal of clinical investigation. 2001;107(7):917-23 doi 10.1172/JCl11947.

28. Goldman EH, Chen L, Fu H. Activation of apoptosis signal-regulating kinase 1 by reactive oxygen species through dephosphorylation at serine 967 and 14-3-3 dissociation. The Journal of biological chemistry. 2004;279(11):10442-9 doi 10.1074/jbc.M311129200.

29. Zhang R, He X, Liu W, Lu M, Hsieh JT, Min W. AIP1 mediates TNF-alpha-induced ASK1 activation by facilitating dissociation of ASK1 from its inhibitor 14-3-3. The Journal of clinical investigation. 2003;111(12):1933-43 doi 10.1172/JCI17790.

30. Brenner B, Ilson DH, Minsky BD. Treatment of localized esophageal cancer. Seminars in oncology. 2004;31(4):554-65.

31. Stahl M, Stuschke M, Lehmann N, Meyer HJ, Walz MK, Seeber S, et al. Chemoradiation with and without surgery in patients with locally advanced squamous cell carcinoma of the esophagus. Journal of clinical oncology : official journal of the American Society of Clinical Oncology. 2005;23(10):2310-7 doi 10.1200/JC0.2005.00.034.

32. Wu K, Xie D, Zou Y, Zhang T, Pong RC, Xiao G, et al. The mechanism of DAB2IP in chemoresistance of prostate cancer cells. Clinical cancer research : an official journal of the American Association for Cancer Research. 2013;19(17):4740-9 doi 10.1158/1078-0432.CCR-13-0954.

33. Ichijo H, Nishida E, Irie K, ten Dijke P, Saitoh M, Moriguchi T, et al. Induction of apoptosis by ASK1, a mammalian MAPKKK that activates SAPK/JNK and p38 signaling pathways. Science. 1997;275(5296):90-4.

34. Saitoh M, Nishitoh H, Fujii M, Takeda K, Tobiume K, Sawada Y, et al. Mammalian thioredoxin is a direct inhibitor of apoptosis signal-regulating kinase (ASK) 1. The EMBO journal. 1998;17(9):2596606 doi 10.1093/emboj/17.9.2596.

35. Shiizaki S, Naguro I, Ichijo H. Activation mechanisms of ASK1 in response to various stresses and its significance in intracellular signaling. Advances in biological regulation. 2013;53(1):135-44 doi 
10.1016/j.jbior.2012.09.006.

36. Riley PA. Free radicals in biology: oxidative stress and the effects of ionizing radiation. International journal of radiation biology. 1994;65(1):27-33.

37. Wallace SS. Enzymatic processing of radiation-induced free radical damage in DNA. Radiation research. 1998;150(5 Suppl):S60-79.

38. Dayal R, Singh A, Pandey A, Mishra KP. Reactive oxygen species as mediator of tumor radiosensitivity. Journal of cancer research and therapeutics. 2014;10(4):811-8 doi 10.4103/09731482.146073.

39. Min W, Lin Y, Tang S, Yu L, Zhang H, Wan T, et al. AIP1 recruits phosphatase PP2A to ASK1 in tumor necrosis factor-induced ASK1-JNK activation. Circulation research. 2008;102(7):840-8 doi 10.1161/CIRCRESAHA.107.168153.

\section{Tables}

Table 1 Clinicopathological correlation of DAB2IP expression in ESCC 


\begin{tabular}{|c|c|c|c|}
\hline \multirow[b]{2}{*}{ Variables } & \multicolumn{2}{|c|}{ DAB2IP expression } & \multirow[b]{2}{*}{$P$ value } \\
\hline & High & Low & \\
\hline$\overline{\text { Age } \llbracket \text { years } \square}$ & & & \\
\hline$<=55^{b}$ & $22(24.2 \%)$ & $69(75.8 \%)$ & 0.759 \\
\hline$>55$ & $13(26.5 \%)$ & $36(73.5 \%)$ & \\
\hline Sex & & & 0.187 \\
\hline Male & $19(21.3 \%)$ & $70(78.7 \%)$ & \\
\hline Female & $16(31.4 \%)$ & $35(68.6 \%)$ & \\
\hline Location & & & 0.399 \\
\hline Cervical/UpperThoracic & $9(31.0 \%)$ & $20(69.0 \%)$ & \\
\hline Middle/Lower Thoracic & $26(23.4 \%)$ & $85(76.6 \%)$ & \\
\hline WHO grade & & & $<0.001$ \\
\hline G1 & $21(61.8 \%)$ & $13(38.2 \%)$ & \\
\hline G2 & $9(12.3 \%)$ & $64(87.7 \%)$ & \\
\hline G3 & $5(15.2 \%)$ & $28(84.8 \%)$ & \\
\hline Tumor size $(\mathrm{cm})$ & & & $<0.001$ \\
\hline$<=5^{\mathrm{c}}$ & $23(41.8 \%)$ & $32(58.2 \%)$ & \\
\hline$>5$ & $12(14.1 \%)$ & $73(85.9 \%)$ & \\
\hline $\mathrm{T}$ Status & & & 0.003 \\
\hline $\mathrm{T} 2 / 3$ & $11(14.9 \%)$ & $63(85.1 \%)$ & \\
\hline $\mathrm{T} 4$ & $24(36.4 \%)$ & $42(63.6 \%)$ & \\
\hline $\mathrm{N}$ Status & & & $<0.001$ \\
\hline No & $20(62.5 \%)$ & $12(37.5 \%)$ & \\
\hline N1 & $15(13.9 \%)$ & $93(86.1 \%)$ & \\
\hline M Status & & & 0.017 \\
\hline M0 & $27(32.1 \%)$ & $57(67.9 \%)$ & \\
\hline M1-lym & $8(14.3 \%)$ & $48(85.7 \%)$ & \\
\hline CRT response & & & $<0.001$ \\
\hline $\mathrm{CR}$ & $18(50.0 \%)$ & $18(50.0 \%)$ & \\
\hline Non-CR & $17(16.3 \%)$ & $87(83.7 \%)$ & \\
\hline Locoregional progression & & & 0.049 \\
\hline Present & $10(16.7 \%)$ & $50(83.3 \%)$ & \\
\hline Absent & $25(31.2 \%)$ & $55(68.8 \%)$ & \\
\hline
\end{tabular}

${ }^{\mathrm{a}}$ Chi-squaretest. ${ }^{\mathrm{b}}$ Mean age. ${ }^{\mathrm{c}}$ Mean tumor size.

Abbreviation:T, tumor; $\mathrm{N}$, node; $\mathrm{M}$, metastases; CRT, chemoradiotherapy; CR, complete response; M1-lym, distant lymph node metastasis; ESCC, esophageal squamous cell carcinoma.

Table 2 Predictive variables for OS and DSS of ESCC patients by log rank test 


\begin{tabular}{|c|c|c|c|c|c|c|}
\hline \multirow[b]{2}{*}{$\begin{array}{l}\text { Variables } \\
\text { Age(years) }\end{array}$} & \multicolumn{3}{|c|}{ OS(months) } & \multicolumn{3}{|c|}{ DSS(months) } \\
\hline & Case & Median & $\begin{array}{l}P \\
0.830\end{array}$ & Case & Median & $\begin{array}{l}P^{\mathrm{a}} \\
0.908\end{array}$ \\
\hline$<=55^{b}$ & 91 & 17.10 & & 71 & 15.40 & \\
\hline$>55$ & 49 & 17.80 & & 41 & 17.80 & \\
\hline Gender & & & 0.348 & & & 0.316 \\
\hline Male & 89 & 17.80 & & 69 & 16.70 & \\
\hline Female & 51 & 17.10 & & 43 & 17.10 & \\
\hline Location & & & 0.353 & & & 0.385 \\
\hline Cervical/upper thoracic & 29 & 18.03 & & 24 & 17.80 & \\
\hline Middle/lower thoracic & 111 & 17.30 & & 88 & 15.90 & \\
\hline WHO grade & & & 0.069 & & & 0.013 \\
\hline G1 & 34 & 21.40 & & 26 & 22.40 & \\
\hline G2 & 73 & 18.03 & & 57 & 15.10 & \\
\hline G3 & 33 & 15.70 & & 29 & 15.50 & \\
\hline Tumor size $(\mathrm{cm})$ & & & 0.004 & & & 0.003 \\
\hline$<=5 \mathrm{~cm}^{\mathrm{c}}$ & 55 & 27.10 & & 41 & 28.40 & \\
\hline$>5 \mathrm{~cm}$ & 85 & 14.77 & & 71 & 14.60 & \\
\hline T status & & & $<0.001$ & & & 0.001 \\
\hline $\mathrm{T} 2 / 3$ & 74 & 26.80 & & 64 & 22.60 & \\
\hline $\mathrm{T} 4$ & 66 & 13.70 & & 48 & 12.80 & \\
\hline N status & & & 0.043 & & & 0.028 \\
\hline No & 32 & 22.30 & & 26 & 22.60 & \\
\hline N1 & 108 & 16.90 & & 86 & 15.10 & \\
\hline M status & & & 0.059 & & & 0.020 \\
\hline M0 & 84 & 19.90 & & 63 & 19.30 & \\
\hline M1-lym & 56 & 15.40 & & 49 & 14.80 & \\
\hline CRT response & & & $<0.001$ & & & $<0.001$ \\
\hline CR & 36 & 36.90 & & 31 & 36.90 & \\
\hline Non-CR & 104 & 14.60 & & 81 & 13.90 & \\
\hline DAB2IP expression & & & 0.002 & & & $<0.001$ \\
\hline High & 35 & 28.80 & & 25 & 34.50 & \\
\hline Low & 105 & 15.90 & & 87 & 14.77 & \\
\hline
\end{tabular}

aLog-rank test. bMean age. cMean tumor size.

Abbreviation: OS, overall survival; DSS, disease specific survival.

Table 3 Results of univariate Cox proportional-hazards analysis for DSS for ESCC 


\begin{tabular}{llll}
\hline Variable & $P$-values & Hazard ratios & $95 \% \mathrm{CI}^{\mathrm{a}}$ \\
\hline Age(years) & 0.908 & 0.977 & $0.654-1.458$ \\
Gender & 0.317 & 0.814 & $0.544-1.218$ \\
Location & 0.387 & 0.813 & $0.509-1.299$ \\
WHO grade & 0.012 & 1.407 & $1.077-1.839$ \\
Tumor size $(\mathrm{cm})$ & 0.003 & 1.839 & $1.224-2.764$ \\
T status & 0.001 & 1.929 & $1.306-2.849$ \\
N status & 0.030 & 1.670 & $1.051-2.654$ \\
M status & 0.022 & 1.585 & $1.070-2.348$ \\
CRT response & $<0.001$ & 4.569 & $2.752-7.585$ \\
DAB2IP expression & $<0.001$ & 2.836 & $1.708-4.710$ \\
\hline
\end{tabular}

${ }^{\mathrm{a} C I}$, confidence interval.

\section{Supplementary Information}

Additional file 1: Table S1. Cox multivariate analyses of prognostic factors on disease.

Additional file 2: Table S2. Clonogenic survivals were fitted to the linear quadratic model and SER was calculated.

Additional file 3: Supplementary Materials and Methods

Figures 
A

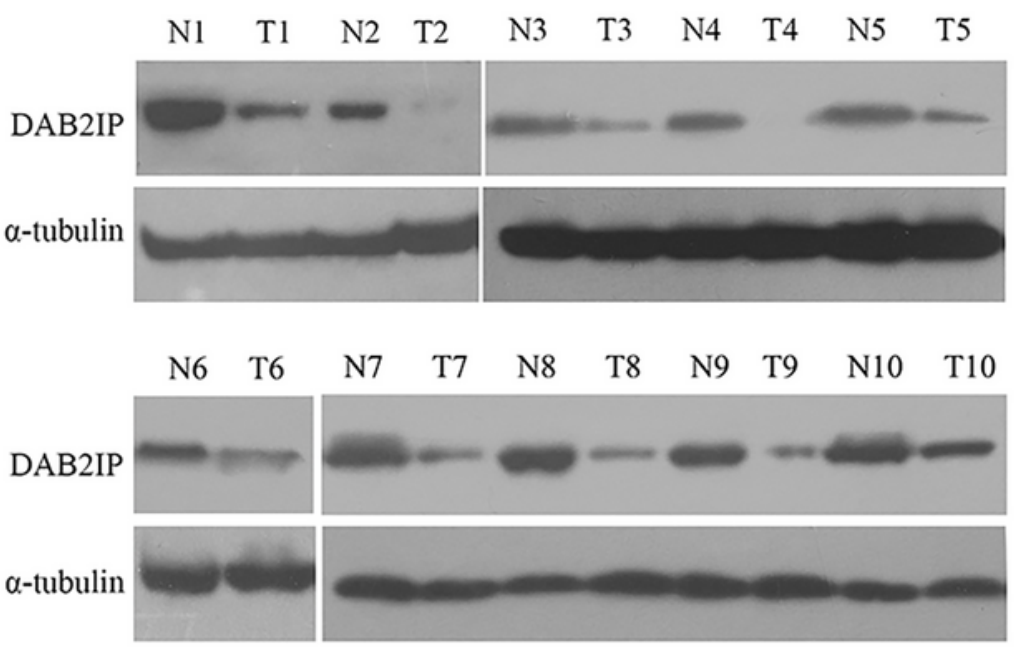

B

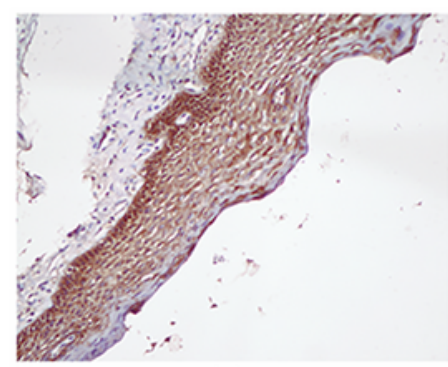

(a)

C

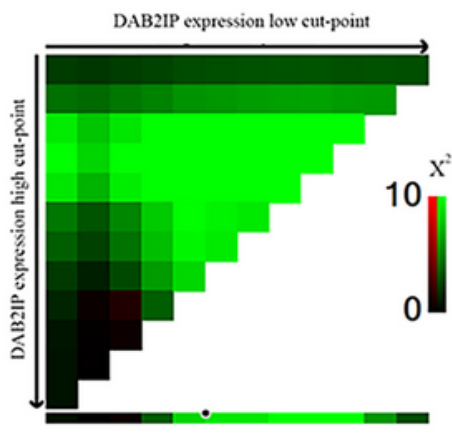

(a)

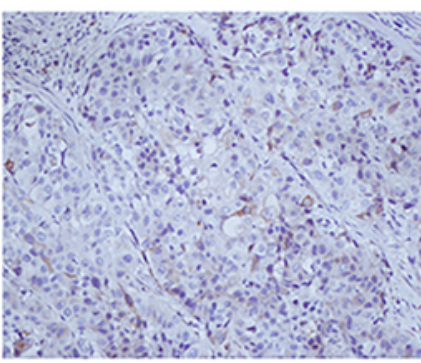

(b)

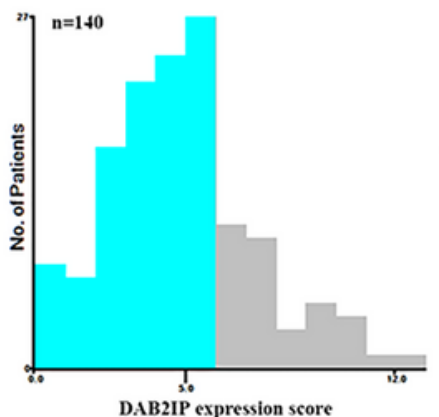

(b)

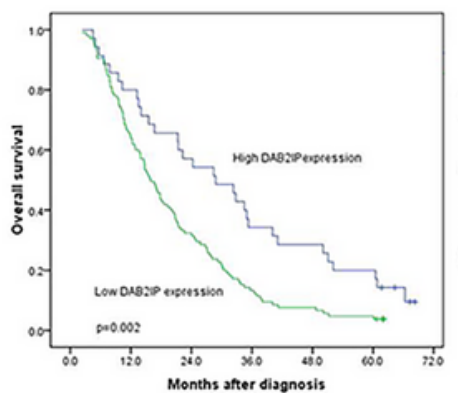

(c)

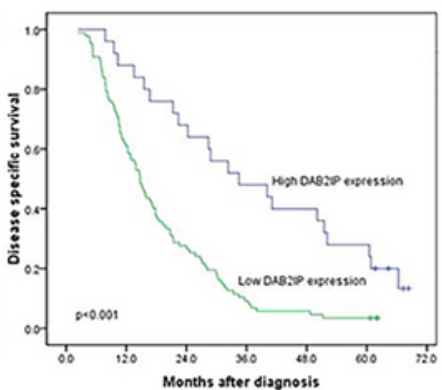

(d)

\section{Figure 1}

Expression of DAB2IP in ESCC cell lines and tissues and its prognostic significance in ESCC patients. (A)The levels of DAB2IP protein in four ESCC cell lines and normal esophageal mucosa (N1 and N2) examined by Western blot (left); Western blot analysis of DAB2IP protein expression in the 10representative paired of ESCC $(\mathrm{T})$ and normal esophageal mucosa (N) (right).(B)(a)Representative images showed strong DAB2IP IHC signaling of normal esophageal mucosa.(b)Low DAB2IP expression 
was detected in ESCC case \#32 (total score=1+1), with $15 \%$ (staining percentage score=1) carcinoma cells demonstrated weak staining (staining intensity score $=1$ ) of DAB2IP.(c)Moderate DAB2IP expression (total score $=6+2$ ) in ESCC case \#45, where about 60\%-70\% (staining percentage score $=6$ ) carcinoma cells demonstrated moderate staining (staining intensity score=2) of DAB2IP.(d) High expression (total score $=9+3$ ) of DAB2IP was observed in ESCC case \#91, with 100\% (staining percentage score=9) carcinoma cells demonstrated strong staining (staining intensity score=3) of DAB2IP.(C) X-tile plots of the prognostic marker DAB2IP. X-tile analysis was conducted on patient data from our ESCC cohort. The plot showed the $\chi 2$ log-rank values created when the cohort was divided into two population. (a)The cut point highlighted by the black/white circle was demonstrated on the histogram. (b)DAB2IP expression was divided at the optimal cut point, as defined by the most significant on the plot. (c, d) Kaplan-Meier survival analysis of DAB2IP expression for OS $(n=140,(c))$ and DSS $(n=112$, (d)) in ESCC patients treated with definitive CRT (log-rank test). 
A
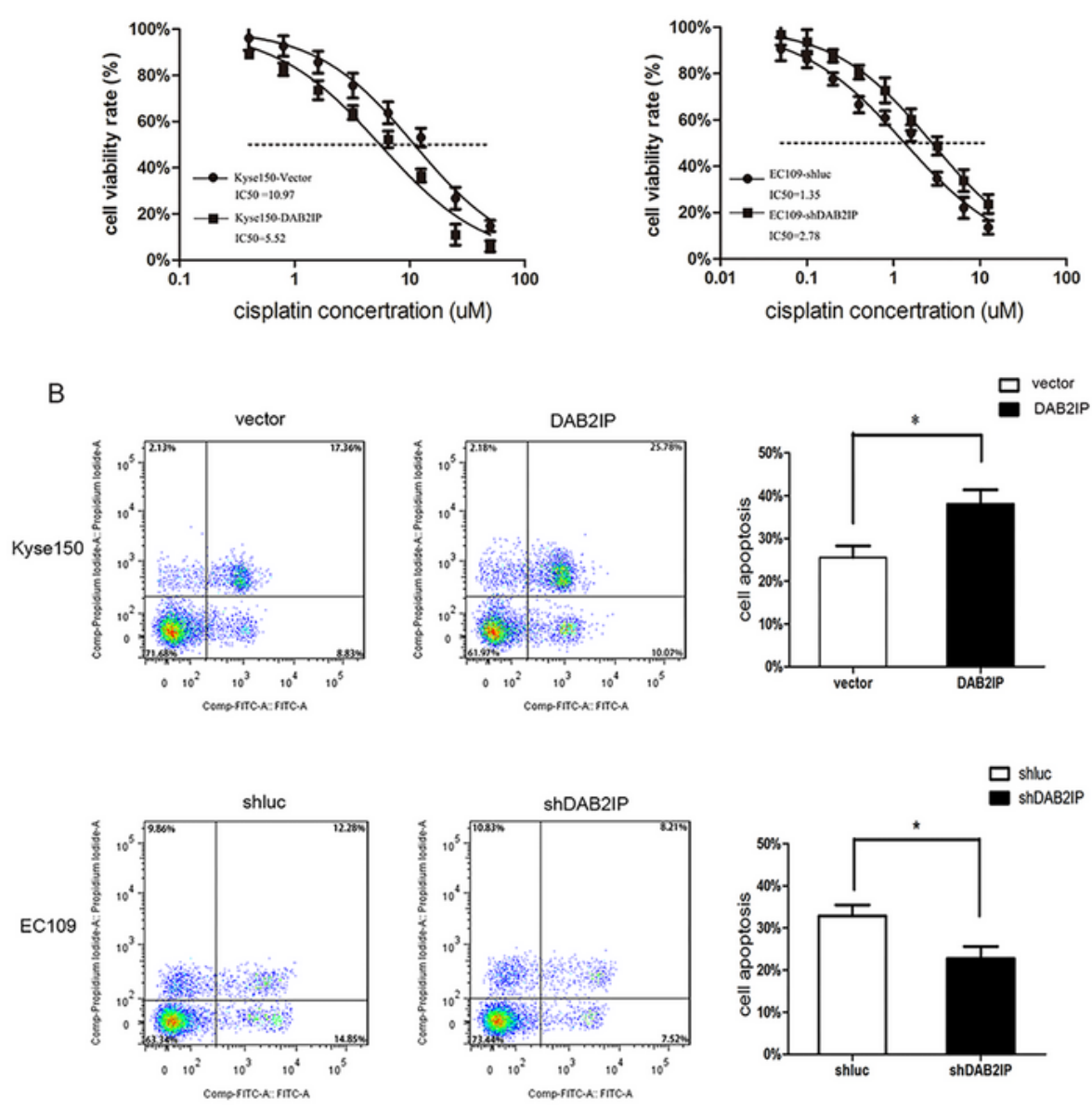

C
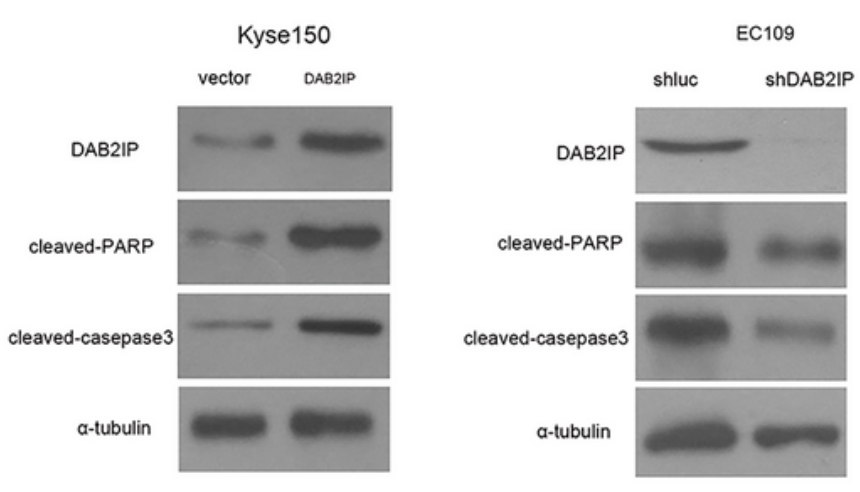

\section{Figure 2}

The impact of DAB2IP levels on the chemosensitivity of ESCC cells in vitro. (A) Shift of dose-response curves of cisplatin by overexpression or knockdown of DAB2IP was determined by MTS assay. Overexpression of DAB2IP in Kyse150 cells is significantly more sensitive to cisplatin than parental control cells (left). Consistently, knockdown of DAB2IP reduced sensitivity of EC109 cells to cisplatin (right).IC50 values were shown below. (B) Upper panels, Kyse150-DAB2IP and the control Kyse150- 
vectorcells were treated with cisplatin (IC30), and 24 hours later, the cells were collected and stained with PI and AnnexinV. Lower panels, EC109-shluc and EC109-shDAB2IP cells were incubated with cisplatin (IC30). After 24 hours, Annexin V and PI staining assay was used to determine the percentage of cells undergoing apoptosis. The $p$-values were calculated by the Student's $t$ test. The bar shows the mean \pm SD of three independent experiments. ${ }^{*} p<0.05$. (C) Left, 24 hours after cisplatin (IC30) treatment, overexpression of DAB2IP increased the levels of cleaved PARP and cleaved caspase-3 in Kyse150 cells. Right, silencing of DAB2IP decreased cisplatin-induced levels of cleaved PARP and cleaved caspase-3 in EC109 cells. 
A
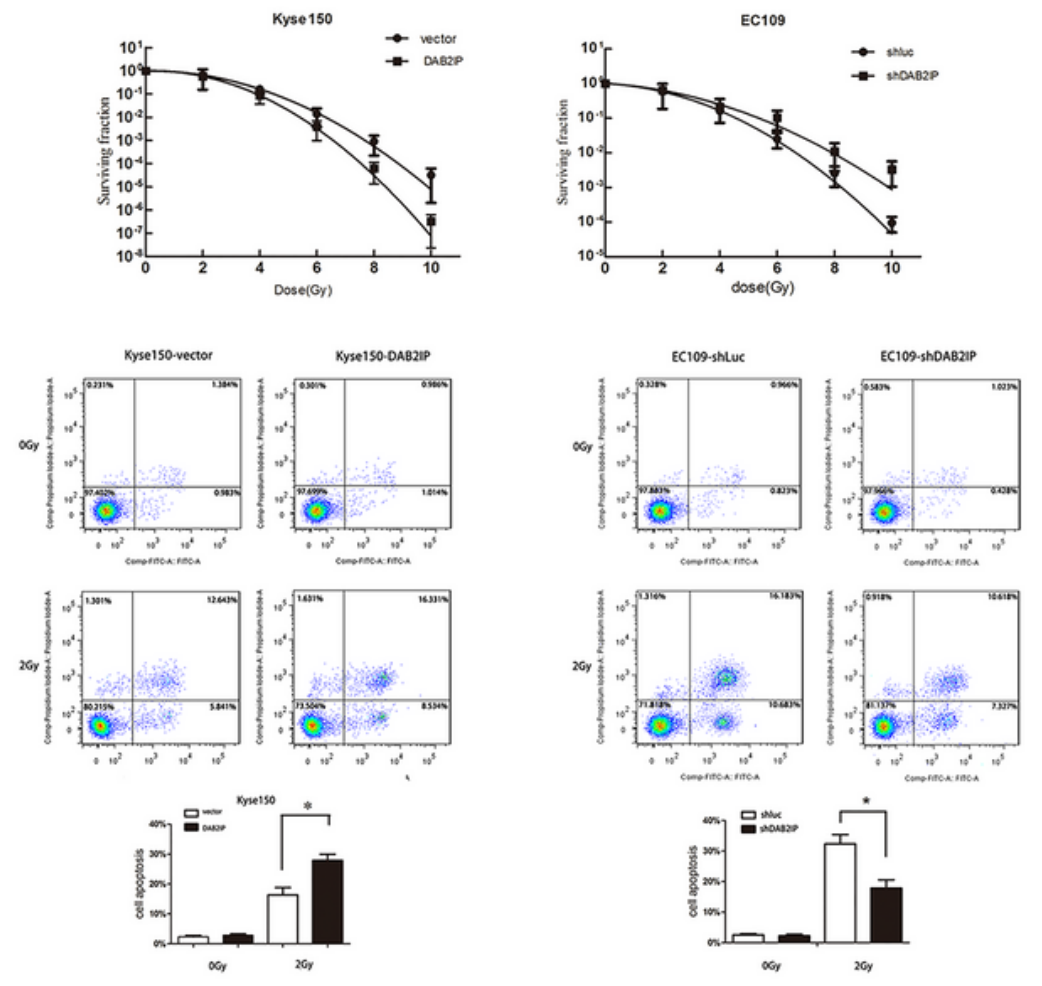

c
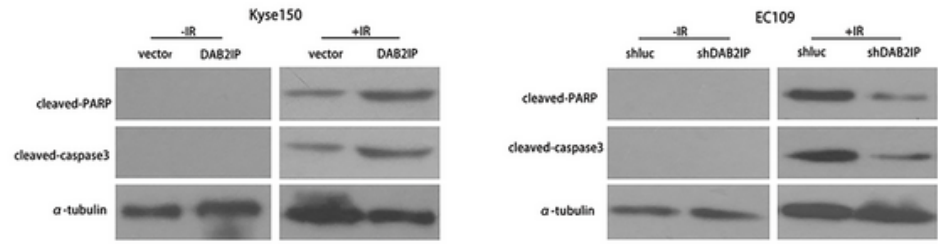

D
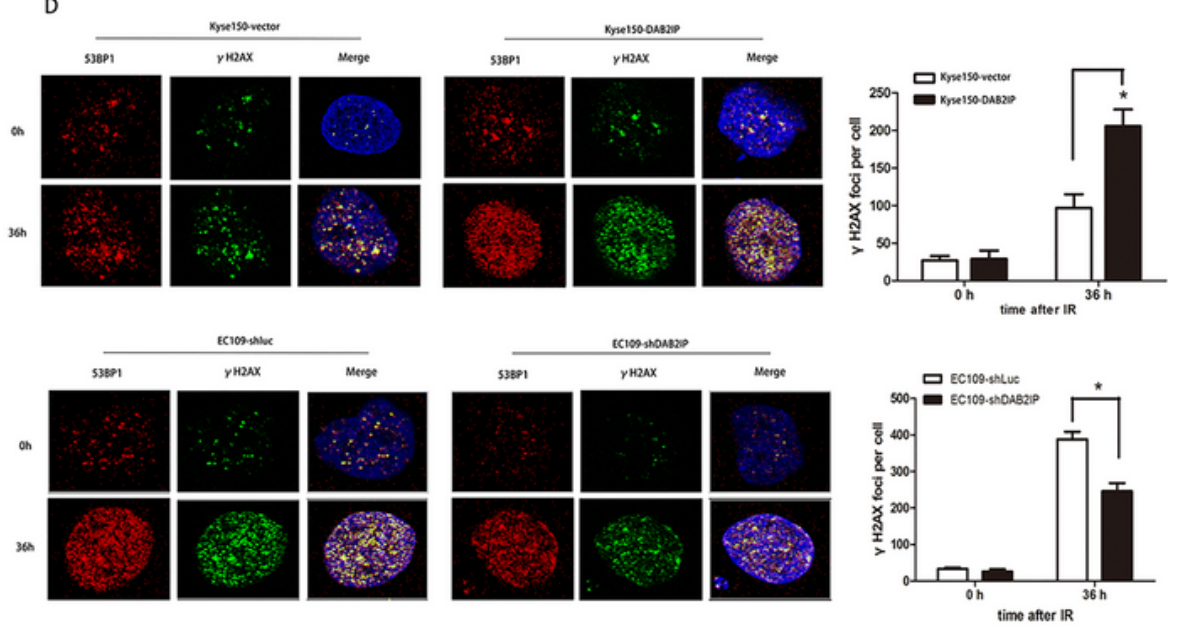

\section{Figure 3}

The expression levels of DAB2IP modulate the radiosensitivity of ESCC cells in vitro. (A) Left, clonogenic survivals of DAB2IP overexpressed cells of Kyse150 cell lines were reduced compared to parental cells after a single dose radiation (0, 2, 4, 6, 8 and 10Gy). Right, knockdown of DAB2IP increased clonogenic survival fraction of EC109. All data were from three independent experiments, and were fitted according to the linear quadratic mode. (B) Left panels, Kyse150-DAB2IP and control Kyse150-vector cells were 
treated with or without IR (3Gy), and 48 hours later, the cell apoptotic death events were monitored by Annexin V/PI staining cells. Right panels, EC109 cells, initially infected with DAB2IP-shRNA and LucshRNA, were treated with or without IR. After 48 hours, Annexin V/PI staining was used to determine the percentage of cells undergoing apoptosis. All data described are the mean \pm SD of three independent experiments. ${ }^{*} p<0.05$. All data are reported as mean $\pm S D$. (C) Forty-eight hours after the cells were treated with IR (3Gy), the cleavages PARP and cleaved casepase-3 were detected in Kyse150vector/Kyse150-DAB2IP (left) and EC109-shluc/EC109-shDAB2IP (right) cells by Western blot analysis. Experiments were performed three times and a representative result is shown. (D) The levels of DAB2IP affect IR-induced DNA damage in ESCC cells. Kyse150-vector/Kyse150-DAB2IP (upper panels) and EC109-shluc/EC109-shDAB2IP (lower panels) were irradiated with 3Gy and immunostained for 53BP1 (red) and phospho-yH2AX (green) foci at the indicated time points after radiation. Colocalized foci (yellow) were used as a measure of DSB. Nuclei were counterstained with DAPI (blue). Columns (right) indicate quantification of average numbers of IR-induced colocalized foci per cell ( 0 or $36 \mathrm{~h}$ after IR). A total of 100 cells from three independent experiments were counted for each group. The data represent mean $\pm S D$. ( ${ }^{*} p<0.05$, $p$-value was according to Student's $t$ test).

\section{Figure 4}

Upregulated expression of DAB2IP enhances the killing-effect of IR on ESCC cell xenografts. (A) Kyse150DAB2IP or control Kyse150-vectorcells (106) were injected subcutaneously into right flank of athymic nude mice, respectively. When the volume of a transplanted tumor grew to $180 \mathrm{~mm} 3$, the tumor were received $6 \mathrm{~Gy}$ dose of IR. The tumor volume of xenografts was measured with calipers every 3 days for a total of 33 days. Overexpressed DAB2IP alone did not affect the growth of the tumors; the mean tumor volume in the Kyse150-vectorand Kyse150-DAB2IP groups was $936 \pm 68 \mathrm{~mm} 3$ and $989 \pm 76 \mathrm{~mm} 3$, respectively ( $n=7, p=0.1943$, Student's ttest). However, when treated transplanted tumor with $6 \mathrm{~Gy}$ dose of IR, the mean tumor volume of the Kyse150-vectorgroup was significantly larger than that of the Kyse150DAB2IP group ( $423 \pm 43 \mathrm{~mm} 3$ vs $287 \pm 38 \mathrm{~mm} 3 \llbracket p \leq 0.05)$. The values represent mean tumor volume $\pm S D$. (B) Representative images of DAB2IP IHC staining in Kyse150-vector and Kyse150-DAB2IP transplanted tumor tissues. (C) Nude mice xenograft from Kyse150-vector and Kyse150-DAB2IP cells were treated with $6 \mathrm{~Gy}$ dose of IR, and 0 or 24 hours later, immunofluorescence with antibodies with $\mathrm{yH} 2 \mathrm{AX}$ (green) and 53BP1 (red) was performed. Merged spots (yellow) show colocalization of 53BP1 and YH2AX foci at DSBs. Experiments were performed three times and a representative result is shown. 
A

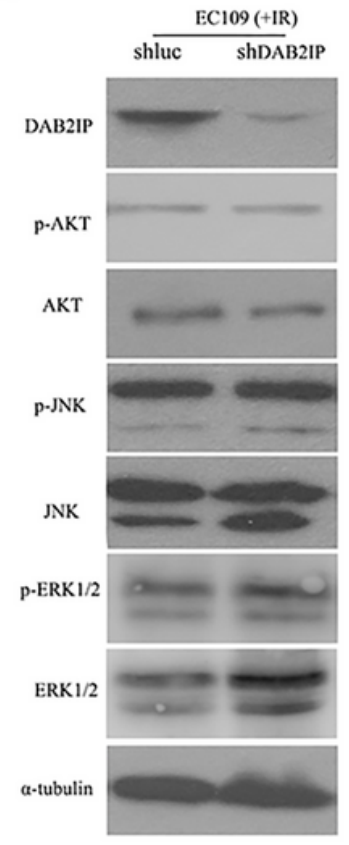

D

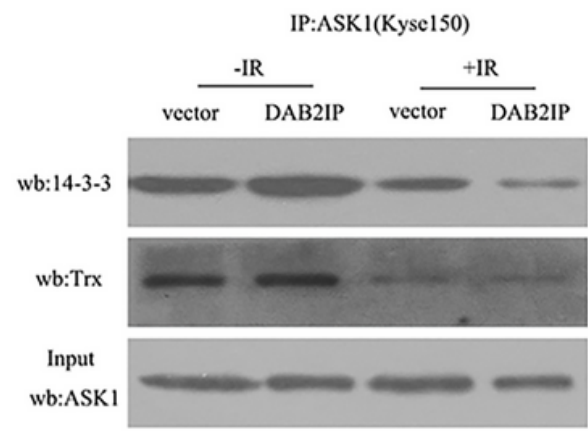

B

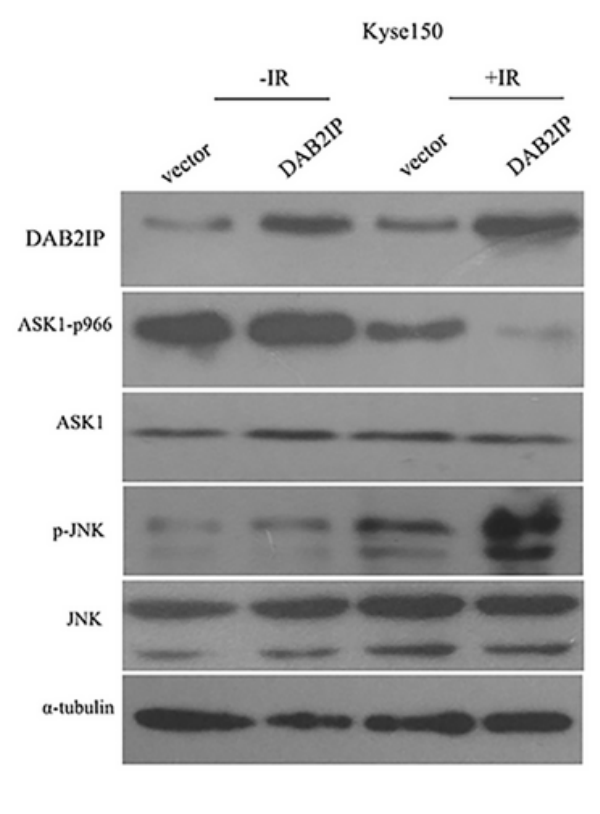

E

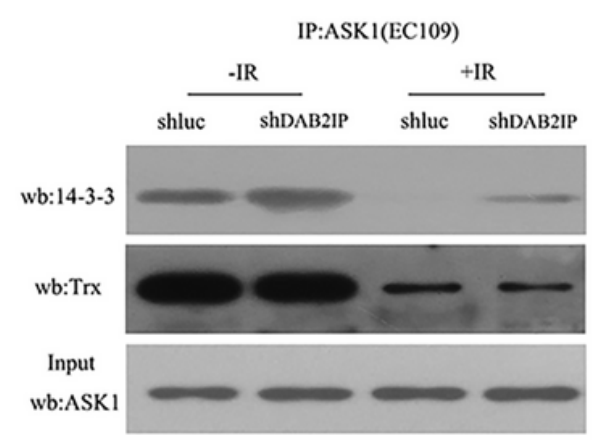

C
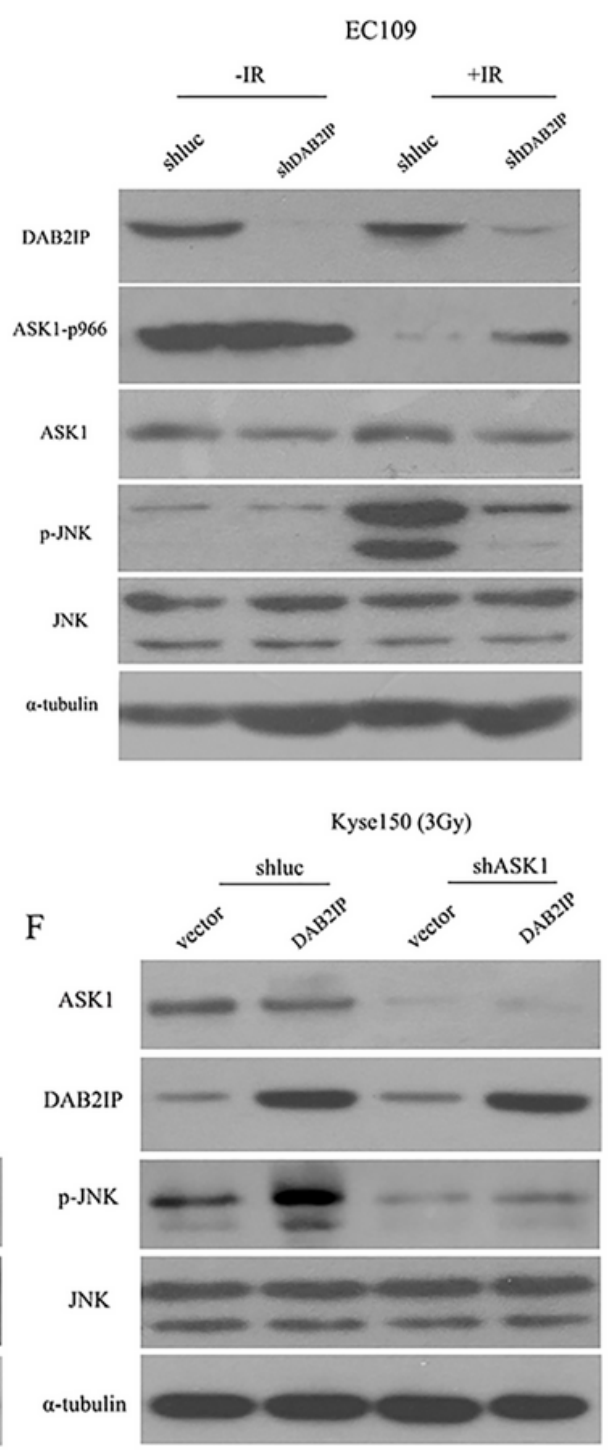

\section{Figure 5}

The critical role of DAB2IP in modulating IR-induced ASK1-JNK pathway activation. (A) EC109-shluc and EC109-shDAB2IP cells were initially treated with 3Gy dose of IR, and 36 hours later, the indicated protein levels were evaluated by Western blotting. Silencing DAB2IP by lentivirus obviously increased the protein levels of p-JNK. No changes were observed in p-ERK and p-AKT levels. (B)DAB2IP enhanced IR-induced dephosphorylation of ASK1-pSer966 and JNK activation.Kyse150 cells were initially transfected withpcDNA3.1-DAB2IP or vehicle pcDNA3.1 plasmid, followed by treatment with or without IR, and 36 hours later, ASK1-pSer966, and JNK activation were measured by Western blotting. (C)EC109 cells were firstly infected with DAB2IP-shRNA or control luc-shRNA lentivirus, and subsequently received with or without IR. After 36 hours, Western blotting was performed to detect levels of ASK1-pSer966 and p-JNK. Results are representative data of three independent experiments. (D)DAB2IP enhanced IR-induced 
disruption of ASK1-14-3-3 complex, but not ASK1-Trx complex. Kyse150 cells were initially transfected with pcDNA3.1-DAB2IP or vehicle pcDNA3.1 plasmid, followed by treatment with or without IR, and 36 hours later, cell extracts were firstly immunoprecipitated by anti-ASK1 antibody, and then the precipitate was immunoblotted(IB) with 14-3-3 and Trx antibody. Input was equivalent to $30 \%$ of the cell lysate used in the immunoprecipitation. (E)EC109 cells were initially infected with lentivirus carried with DAB2IPshRNA or luc-shRNA, subsequently received with or without IR. After 36 hours, interaction of ASK1 with endogenous 14-3-3 or Trx was determined by coimmunoprecipitation with primary anti-ASK1 antibody followed by Western blot with 14-3-3 or Trx antibody. The input represents $30 \%$ of the cell lysates. Each immunoprecipitation experiment was repeated at least three times and a representative experiment is shown. (F) The enhanced activity of DAB2IP on IR-activated JNK signal was dependent on the presence of ASK1. The Kyse150 cells were firstly infected with ASK1-shRNA or control luc-shRNA lentivirus, and then each group cells were transfected with pcDNA3.1-DAB2IP or vehicle pcDNA3.1 plasmid. All group cells were followed by 3Gy IR treatment, and 36 hours later, JNK activation were evaluated by Western blotting with p-JNK antibody. The representative blots of three independent experiments were shown.

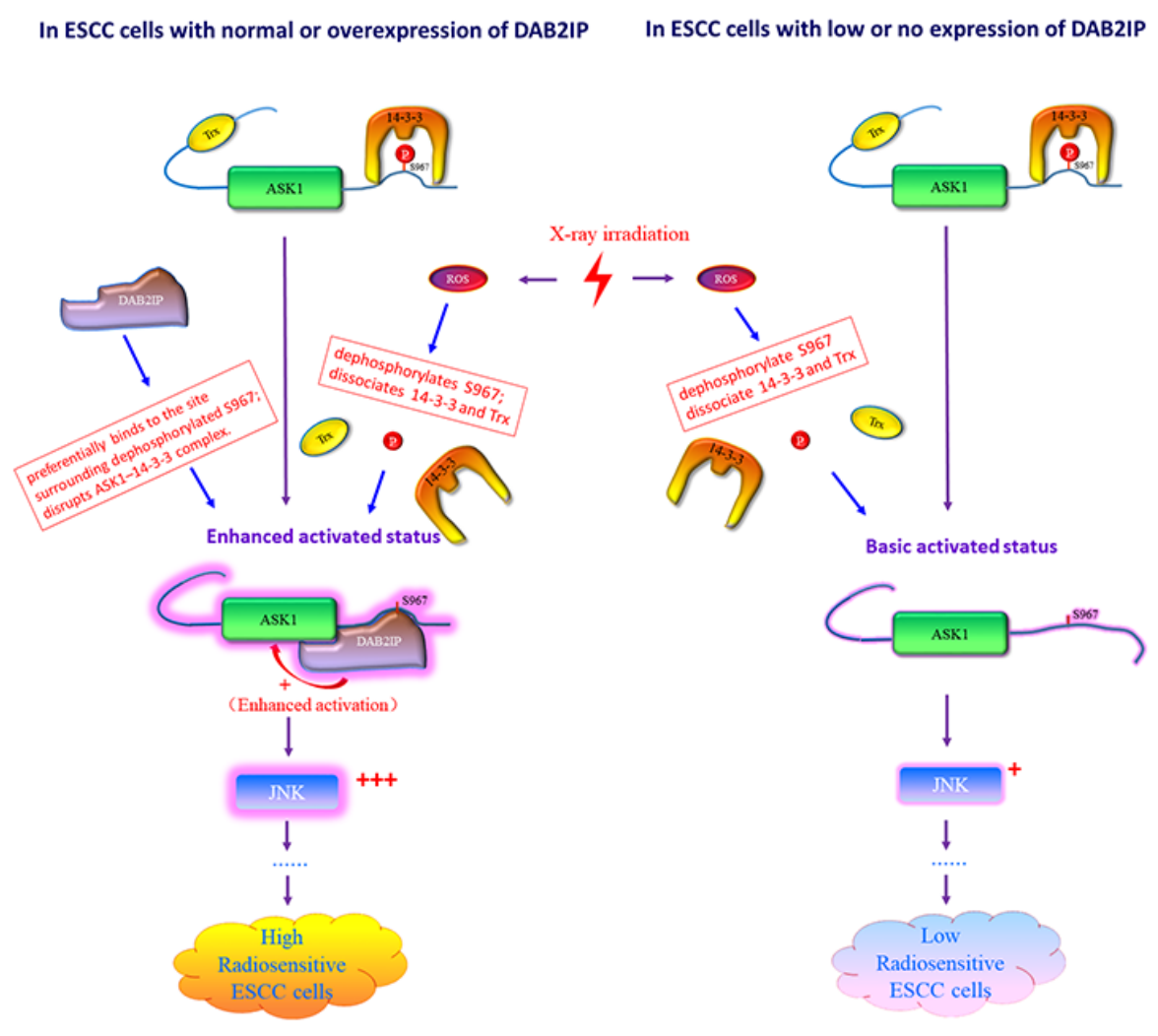

\section{Figure 6}

Proposed schematic representation for the molecular mechanism of DAB2IP-induced regulation of radiosensitivity in ESCC cells. The detailed explanation was described in discussion paragraph 9. 


\section{Supplementary Files}

This is a list of supplementary files associated with this preprint. Click to download.

- SupplementaryTable2.pdf

- SupplementaryMaterials.pdf

- SupplementaryTable2.pdf

- SupplementaryMaterials.pdf

- SupplementaryTable1.pdf

- SupplementaryTable1.pdf 